\title{
Concealing paradoxes in decision-making during hospital hybridization - A systems theoretical analysis
}

\begin{abstract}
Purpose - While previous healthcare-related hybridity research has focused on macro and micro level investigations, in this paper we study hybridization at the organizational level with a specific focus on decision-making. We investigate how new politico-economic expectations towards a university hospital as a hybrid organization become internalized via organizational decisionmaking, resulting in the establishment of a new business collaboration and innovation oriented unit.
\end{abstract}

Design/methodology/approach - We employed a social systems theoretical framework to explore organizational decision-making processes involved in the establishment of the new hybrid hospital unit. Drawing on 15 interviews and nine organizational documents, we describe and analyze three decision-making cycles using the concepts of complexity, decision and justification.

Findings - The findings reveal the challenging nature of decision-making during hybridization as decisions regarding unprecedented organizational structures and activities cannot be justified by traditional decision premises. We show that decision-makers use a combination of novel justification strategies, namely justification by problems, by examples and by obligations, to legitimize decisions oriented at non-traditional activities. Further, the analysis reveals how expectations of several societal systems, i.e. healthcare, education, science, law, economy and politics, are considered in decision-making taking place in hybrid organizations.

Originality - The study draws attention to the complexity of decision-making in a hybrid context and highlights the role of justification strategies in partially reducing complexity by concealing the paradoxical nature of decision-making and ensuring the credibility of resulting decisions. Also, the study presents a move beyond the dualism inherent in many previous hybridity studies by illustrating the involvement of several societal systems in hybridization.

Keywords: organizational hybridity, decision-making, healthcare, social systems theory

Paper type: Research paper

Jansson, K., Tuunainen, J. and Mainela, T. (2021), "Concealing paradoxes in decision-making during hospital hybridization - a systems theoretical analysis", Journal of Health Organization and Management, Vol. 35 No. 2, pp. 195-211. https://doi.org/10.1108/JHOM-08-2020-0334 


\section{Introduction}

Over the last decade, hybridity and hybrid organizations have received increasing attention from organizational and public administration scholars. Most prominently, organizational research has used an institutional logics approach (Battilana et al., 2017) to study social enterprises combining market and social welfare logics (Pache and Santos, 2013; Battilana and Lee, 2014), but hybridity has also been studied in such diverse contexts as research institutes (Gulbrandsen, 2011), the third sector (Brandsen et al., 2005) and healthcare (Powell and Castelli, 2017). In public administration, the concept of hybridity is still considered under-developed and is often explored at a macro level by concentrating on formal structures and governance, thus neglecting the local implications of hybridity for organizations, teams and individuals (Denis et al., 2015).

In the present study, the focus is on hybridity in healthcare. In this context, hybridity has been examined in terms of regulatory hybridity in governance (McDermott et al., 2015) and with reference to the hybrid roles, identities and experiences of healthcare professionals (Bjyrkeflot and Jespersen, 2014; Croft et al., 2015; Spyridonidis et al., 2015). Because investigations of hybrid healthcare organizations have thus far been limited, scholars have called for the incorporation of theoretical lenses from organizational studies to increase the multi-level understanding of hybridity in public services and especially in healthcare organizations (Denis et al., 2015; Powell and Castelli, 2017). We argue that while this research has explored a range of management strategies and their implications for hybrid organizations, decision-making in hybrid organizations has not yet received much attention. Additionally, while previous research has discussed the paradoxical nature of hybrids due to the involvement of contrasting logics (Jay, 2013), we suggest that paradoxes are an intrinsic part of organizational decision-making and investigate how they are navigated during hybridization.

Therefore, we apply a promising social systems theoretical approach (Luhmann, 1995; Seidl and Becker, 2006a) to analyze how hybridization manifests in managerial decision-making in a university hospital. We conceptualize hybridity as increasing organizational complexity in the face of emergent societal expectations and investigate decision-making under such conditions. The research question of the study is: How are decisions justified in organizational decision-making during hybridization of a university hospital? 
Our analysis focuses on decision-making involved in setting up a novel testing and innovation unit within a university hospital. The unit was established to initiate and coordinate product development collaborations with healthcare and medical technology businesses, to support the hospital's renewal project and to enhance an internal innovation culture. The analysis draws on Niklas Luhmann's systems theory and its functional method (Besio and Pronzini, 2011; Knudsen, 2011) to generate theoretically-informed observations on the management of paradoxes during the decision-making process.

The findings depict the challenging nature of decision-making during hybridization, as decisions oriented at unprecedented organizational structures cannot be justified by the organization's existing premises for decision-making, such as formal strategies or rules. As its main contribution, the study identifies three justification strategies developed for managing the paradox of decisions, i.e. the fact that each decision could also be made differently, during the decision-making process, named as justification by problems, by examples and by obligations. By concealing the paradoxical nature of decision-making and enhancing acceptability and legitimacy of decisions, these strategies partially reduce complexity. Furthermore, while previous research has investigated tensions arising from dual institutional logics within a single organization, the present study moves beyond dualism by revealing the interaction between multiple societal systems in hybridization of a university hospital.

\section{Hybrids in healthcare and organizational studies}

Hybridity is not a new phenomenon in the public sector and is growing in importance as the sector increasingly blurs with the private and third sectors, healthcare being no exception (Denis et al., 2015). In hospital settings, studies have reported on adoption and implementation of a hybrid practice combining business-like and professional logics (Broek et al., 2014) and hybrid management (Byrkjeflot and Jespersen, 2014). McDermott et al. (2015) examined hybridity in healthcare with a focus on regulatory hybridity, i.e. combining top-down deterrence and bottomup persuasive approaches in system governance. Waring (2015) studied professionals' experiences of cultural hybridity when moving from a public to a private organization, and others have looked at the hybrid roles and identities of healthcare professionals (Croft et al., 2015; Spyridonidis, 2015). A wide range of healthcare organizations have been labeled as hybrids, theorized mostly from cultural, transaction cost economics and governance perspectives, but due to the heterogeneity of 
the limited number of studies, consensus about the definition of a hybrid organization has not been reached (Powell and Castelli, 2017).

The institutional logics approach has reached an established position in hybridity research within organizational studies and is also promoted as potential for advancing hybridity research in public services (Denis et al., 2015; Gestel et al. 2020; Skelcher and Smith, 2015). According to this approach, society is composed of central institutions (DiMaggio, 1991; Friedland and Alford, 1991) that provide distinct logics, defined as socially constructed sets of material practices, assumptions, values and beliefs, that shape cognition and behavior (Thornton et al., 2012). Hybrid organizations are considered to enact elements from multiple of these logics in their operations (Battilana and Dorado, 2010; Skelcher and Smith, 2015).

Most of the hybrid literature using the institutional logics approach has focused on social enterprises (Battilana and Lee, 2014) caught between the competing demands of market and social welfare logics (Pache and Santos, 2013), and scholars have investigated how such contrasting demands are dealt with. Organizations have been found to respond to competing demands by compromise, avoidance, defiance or manipulation (Pache and Santos, 2010), selectively coupling elements of each logic to gain legitimacy (Pache and Santos, 2013) and balancing these by creating a common identity though socialization and hiring practices (Battilana and Dorado, 2010). The plurality of logics and organizations' responses to it can also lead to prioritizing one logic over the other(s), oscillation between these or a novel synthesis of logics (Jay, 2013; Mair et al., 2015). Further, the contrasting logics are considered to make hybrid organizations paradoxical, as actions can be successes by one logic and failures by another, triggering organizational sensemaking (Jay, 2013).

What remains unexplored, however, is how hybridity manifests on the organizational decisionmaking level, and how paradoxicality is handled in decision-making regarding which actions to take in the first place. Therefore, we focus in this paper on organizational decision-making during hybridization by applying a Luhmannian systems theoretical perspective (Luhmann, 1995; 2018) that views paradoxicality as an intrinsic part of all decision-making.

\section{Systems theoretical approach to organizational hybridity}

\subsection{University hospital in a functionally differentiated society}


According to systems theory, modern society is differentiated into autonomous function systems, such as healthcare, law and economy, that serve different societal functions (Luhmann, 1995, Seidl, 2006). The empirical reality of function systems can be observed via organizations, which are another type of social systems, that can localize decisions relevant for the function systems (Nassehi, 2005). Both types of systems are operationally closed, meaning that they do not communicate directly with one another but observe each other from the point of view of problems that are pertinent to their operations (Luhmann, 1986).

The linkages between an organization and function systems, are achieved by structural couplings (Stichweh, 1999; Knudsen, 2007). This means that the organization adjusts its structures to fit the structures of function systems, thus allowing the organizations to react to observed changes in the environment (Seidl, 2006). Changes in the environment stimulate organizations to respond to new environmental complexity by developing novel structural arrangements and thus increasing their internal complexity (Schneider et al., 2017). For example, an embedded intermediary organization designed to connect healthcare and other sectors of society (Singh and Butler, 2015), could be established to reduce complexity, as in the empirical case of the present study. Hence, each structure of an organization can be viewed as a solution to a specific problem of complexity (Seidl and Mormann, 2015).

Aligned with the theory described above, a university hospital is conceptualized as a tri-focal organization primarily coupling the function systems of healthcare, education and science in its internal structures. However, the environment of a hospital is also rife with signals emerging from economic, political, and legal function systems, which must be processed into workable arrangements within the hospital (Knudsen and Vogd, 2015). The hospital observes and makes sense of these communications through its managerial operations (Knudsen, 2007). Politicoeconomic expectations about contributions to healthcare, to medical technology product development and to regional socio-economic development reflect environmental complexity that the hospital seeks to reduce in its decision-making. This is achieved by forming a suborganizational unit that acts as structural coupling between the hospital and the economic and political function systems (Hernes and Bakken, 2003).

\subsection{Justification in organizational decision-making}


Organizations are also communication systems, relying on recursively-connected decision communications (Seidl, 2006; Seidl and Becker, 2006b). In systems theory, a decision means not merely a choice, but a choice oriented at an alternative: in addition to a selected alternative, a decision also contains information about a set of rejected alternatives (Luhmann, 1995; 2018). This creates a paradox, as a decision always communicates the fact that it could have been made otherwise (Knudsen, 2006). Thus, although decisions function to absorb complexity, they also produce more contingency within the organization (Andersen and Pors, 2017). The paradox of a decision threatens the organization's recursive connecting of new decisions to previous ones: Why would a decision be connected to the preceding one if that could have been made otherwise? As paradoxes are unsolvable, they must be managed in organizational decision-making by what is called deparadoxization, meaning hiding the paradoxical nature of each decision to ensure that decision-making is not blocked (Knudsen, 2006). For the purpose of simplicity, we use the term justification hereafter when referring to deparadoxization, focusing on the reasons given for a decision to hide the fact that it could be made differently and thus enhance the decision's acceptability and legitimacy.

Justification can take place via decision premises, meaning that the paradox of the decision is concealed by the decision's premises (Luhmann, 2018). Every decision, if not ignored as noise, acts as a premise for the ensuing decisions and by so doing connects previous and future decisions to one another (Luhmann, 1995). Organizations have two kinds of far-reaching decision premises: so-called decidable ones that include rules and criteria, personnel recruitment and assignments (positions) as well as communication channels that structure and define decision situations (ibid.); and undecidable ones that refer to organizational culture, meaning our ways of doing things (Luhmann, 2018). The difference between these premises is that the decidable ones can be formally agreed upon; an organization can decide, for example, on the content of formal strategies or action plans setting operational guidelines, while organizational culture is considered out of formal control, i.e. undecidable. In addition to the far-reaching premises, other types of justifications may develop (Knudsen, 2006). This paper highlights how hybridization may lead to the development of new justification strategies, as the hospital lacks existing, far-reaching decision premises for decisions regarding unprecedented organizational structures.

\subsection{Theoretical framework of the study}


Drawing on the systems theoretical perspective adopted, we propose that organizational hybridity involves internalization of environmental complexity. This can be achieved via decision-making that couples the structural components of the organization to broader function systems operating in its environment. In the empirical case presented below, the hospital couples with the economic and political function systems through establishing a new sub-organizational unit, referred to hereafter as the testing unit, adjusting the hospital's internal structures to fit new politico-economic expectations observed in the environment. We analyze the decision-making process during this hybridization and, drawing inspiration from Knudsen's (2006) analysis of Danish public sector reforms, focus on how the organization hides the paradoxes inherent in its decisions. Figure 1 illustrates the theoretical framework applied.

< insert: Figure I. Theoretical framework. >

The figure visualizes the tri-focal university hospital organization, primarily coupling healthcare, education and science, and the new testing unit coupling to the economic and political function systems. The function system of law is also included in the figure as the analysis reveals how its expectations, together with the other function systems, also influenced the decision-making process. Decision-making is visualized at the center of the figure; complexity is processed via justification into complexity-reducing decisions, which lead to further complexity in a cyclical manner.

\section{Data and methods}

We investigated decision-making resulting in the emergence of the novel testing unit that was conceived as an embedded intermediary department that would enable the co-development of healthcare innovations by the hospital and its industrial partners. It is formally an official profit unit of the hospital district which manages the entire regional healthcare system and it is mandated to connect the university hospital to external innovative and entrepreneurial activities, feed these into the hospital, and to develop overall innovation culture within the hospital.

The unit's development started from collaborative activities within a new regional health ecosystem bringing together representatives from the university hospital, the city, local higher education and research institutes and businesses. It is connected to the hospital's renewal project, which includes building new facilities and modernization of the hospital structures and operations, 
coordinates and implements innovation collaboration with healthcare and medical technology firms and offers prototype testing services for businesses.

The data covers the decision-making process from the testing unit's planning through the formal establishment and setting up of its activities until its early operations during the five-year period (2013-2018). Fifteen interviews were conducted in 2018-2020 with hospital personnel (9) and members of the local health ecosystem (6). The hospital interviewees were purposively selected to include people who had decision-making power, influence or a close view into the decision-making process via their organizational positions. The interviews lasted between 40 and 150 minutes, resulting in 16 hours and 52 minutes of recordings in total. All recordings were transcribed verbatim afterwards, amounting to 148 pages of text. Additionally, various archival data were collected, including presentation materials, meeting memos, funding applications and reports about the unit's planning, establishment and initial operations. From the archival data, nine documents which directly dealt with the testing unit were chosen to be subjected to detailed analysis. Furthermore, meeting memos of the health ecosystem and the unit's project group were used as background material for tracking events over time.

We used the functional method as the analytical strategy. It allows for identifying and specifying problems arising from complexity and resulting structural solutions adopted by the organization (Seidl and Mormann, 2015), generating observations using the 'problem/solution' distinction (Knudsen, 2011). Thus, when investigating decision-making from the functionalist perspective, each decision is analyzed in terms of the problem it solves (Besio and Pronzini, 2011), and as decisions also produce contingency within the organization (Andersen and Pors, 2017) they create further problems to be solved. The analysis thus developed in a chain of problem/solution/consequent problem/solution (Knudsen, 2011).

The analysis proceeded in three steps. First, the data were read through and a general case description was written. This step identified the sequence of events over time leading to the establishment of the testing unit. Second, the process was examined from the functionalist perspective, identifying descriptions of problems observed by decision-makers and of solutions developed for each problem. However, how the problems, i.e. complexity, were processed in decision-making into solutions, i.e. complexity-absorbing decisions, was not yet revealed. This was achieved in the third stage of the analysis by coding the data using the concepts of complexity, 
justification and decision. In this stage, the decision-making process was also divided into three cycles. The nature of the problems identified in the previous stage of analysis was classified as either related to environmental complexity or to hospital's internal complexity. The descriptions of solutions in turn were detailed into specific complexity-absorbing decisions. Data coded as justification included descriptions of the ways decisions were being argued for in order to hide the paradoxes and enhance their acceptability and legitimacy. This resulted in the identification of three justification strategies that were abductively derived from the data. Hence, following the systems theoretical approach, we searched for descriptions of justification, but the specific strategies described in the next section arose from the empirical data rather than from theory.

\section{Concealing paradoxes in decision-making during a university hospital's hybridization}

The establishment of the testing unit, i.e. a novel organizational structure, in response to observed societal developments introduced challenges for decision-makers. Because the unit and its collaborative activities with businesses were unprecedented, in the beginning of the process there was a lack of existing decision premises, such as the formal strategies, rules or guidelines regarding business collaboration, by which decision-makers could justify decisions regarding the unit. Thus, the decision-makers adopted three justification strategies to conceal the paradoxical nature of decisions and to enhance their acceptability and legitimacy. The decisions reached, however, led to new complexity that had to be addressed in subsequent cycles of decision-making, again demanding the hiding of paradoxes. The findings section is organized around three cycles of decision-making, namely planning, establishment and early operations, analyzing the interplay between complexity, justification strategies and decisions in each cycle.

\subsection{Planning of the testing unit}

\section{Complexity}

The first cycle of the decision-making process started with the hospital management's observation of increasing environmental complexity in the form of new politico-economic expectations in relation to the hospital's contribution to healthcare and medical technology product development. A local health ecosystem had been established by the city's business development enterprise that aimed at developing collaboration between public healthcare and private businesses. By raising the region's attractiveness for businesses and investments, product development collaboration between 
public healthcare and private businesses was expected to bring both economic and social benefits to the region. Businesses had also started to contact the hospital directly regarding problems in gaining access to the hospital. The following interview accounts reflect these politico-economic developments, specifically the need for developing public-private collaboration and increasing the attractiveness of the region:

I asked what the most important thing is to achieve with this ecosystem, and [the previous ecosystem director] ${ }^{1}$ crystallized it as the creation of an operational model for publicprivate collaboration. If we manage to create one, we've succeeded. (Ecosystem director).

(In generally discussing healthcare development ${ }^{2}$ For us here regionally, I'm not sure how much regional politics have to do with it, but at least national politics have a role in that we've always had challenges to attract development projects here instead of the capital city region. So, we have a lot to do on a national level. (Research institute representative, member of the ecosystem)

In addition, hospital's internal complexity ensuing from previous decisions was observed by management. First, internal development processes had advanced to an uncoordinated state. Initiative handling was bureaucratic and slow and many collaborations with businesses were ongoing in the hospital, but often unknowingly of each other. Second, more acute internal complexity ensued from a previous decision to start a renewal project for rebuilding and modernization of the hospital. The renewal project needed facilities for modelling future hospital rooms and procedures for testing solutions to be taken in use in the new premises.

Hence, an idea of a testing unit was developed as a solution to the observed environmental and internal complexities. By offering prototype testing services for businesses and centralizing collaboration, coordinating and developing internal innovation procedures and providing facilities and structures for the renewal project, the new unit would reduce the observed complexity. Due to the lack of existing far-reaching decision premises, like official strategies or guidelines for the planned activities, three emergent justification strategies were developed by decision-makers.

\section{Justification strategies}

The justification by problems strategy meant hiding the paradox of the decision by referring to the problems it would solve. Thus, in this strategy the internal and environmental complexity observed

\footnotetext{
${ }^{1}$ Names are replaced by descriptions in square brackets added by the analyst

${ }^{2}$ Descriptions in brackets added by the analyst for clarity
} 
was considered justification in itself for the decision to establish the testing unit. In a project proposal in 2013, the testing unit was stated to solve the problem of "slow and risky commercialization of healthcare technology" and the following interview extract emphasizes how answering to businesses needs was one of the key reasons given for the testing unit.

We generally received messages saying it was hard for businesses to get into the hospital, the contact is difficult, the process is difficult, and it's difficult to bring in and present any ideas for further development. This was a major issue, so we saw that there was a huge demand for the testing unit because that route was especially difficult (for the businesses). (Hospital's development director)

The unit's establishment was planned via a project with the university of applied sciences and the city in connection to the health ecosystem. In a funding application the project was connected to broad regional benefits. Thus, the decision to establish the testing unit was justified as enhancing socio-economic development of the region, as indicated below.

The [project's] innovation, testing and development environment will benefit the entire social welfare and health care region by providing an opportunity to access the latest medical technology products and systems, which in turn will increase the quality of social welfare and health care services and make the services more affordable. --- Ultimately, the citizens of the region benefit from treatment and health promotion utilizing new products and services. New jobs will be created in the region and thus also tax income will increase. (EU funding application 2014)

The internal complexities also provided justification for the decision, i.e. the internal renewal project's need for facilities and the uncoordinated nature of internal development activities. These aspects are reflected in the accounts below. In the first quote, the hospital manager emphasizes the renewal project as the primary justification for the testing unit, and in the second quote an innovation coordinator describes the internal problems tackled in the testing unit.

In a way, the baseline and the acceptability of the testing unit was based primarily on the renewal of the hospital. So, we were also ready to invest the hospital district's funds in it. (Chief administrative doctor)

If I compare our current innovation activities to what they were before, I must say we've always had innovation activities, but they were scattered, partly overlapping and information didn't flow. They were demanding because of (internal) complexity, the separated nature of medical fields and many other factors that prevented information flow, so we've needed to especially put effort in it. (Innovation coordinator) 
It should also be noted here that referring to the renewal project is also related to the hospital's recursive decision-making. In other words, the previous decision to start the renewal project was used as a premise for the decision to establish the testing unit.

In the second strategy, justification by experience, the paradox of the decision was concealed by emphasizing external and the hospital's own previous experiences of similar activities that were planned for the testing unit. Foreign organizations where private businesses and public healthcare actors co-developed solutions were visited by people involved in the planning and used as justification in decision-making. There were also local examples of business collaboration in one of the city's healthcare centers, in the university of applied sciences and in the university. The account below by a local entrepreneur reflects the role of one of these external examples in providing grounds for the testing unit.

I think (the business collaborations established in the healthcare center) were kind of a turning point, knowing how hard it is to bring the two worlds (of business and healthcare) together. Because we had succeeded so well there, I think afterwards it might have been easy to even copy it, like "well hey, let's do the same thing again". (Healthcare innovation entrepreneur).

Also, the hospital's previous development projects were used as examples. A successful precedent project of building an outpatient facility during which businesses' products and services had been tested, was especially used as justification for the testing unit. This reference to the hospital's own previous projects is also related to recursive decision-making, i.e. previous decisions regarding development projects were used as premises for the decision to establish the unit. Additionally, the hospital's tradition in medical research was emphasized as giving a sound basis for new testing and development activities.

The third strategy is called justification by obligations. It concealed the paradox of the decision by emphasizing the demands of different function systems, especially healthcare, science, education and law, which the hospital had to follow. Thus, via this strategy the decision was justified by its importance for fulfilling the hospital's existing purposes and obligations. For example, the testing unit was stated to increase the number of scientific publications, decrease healthcare costs and enhance quality (Project proposal 2013), and although it would be part of the health ecosystem, it was pressed that it would primarily serve the needs of the hospital district (Hospital's development director's presentation 2014). Hence, the unit would aid in fulfilling the hospital's healthcare and 
scientific purposes. Also, by providing a context for clinical skills training for hospital staff, medical students and specialist doctors under training (Presentation for hospital district board 2014), it would fulfil also the hospital's education purposes. Lastly, the interview extract below illustrates how the testing unit's and its co-development activities were justified by the need to overcome the limitations of procurement legislation that had to be followed in acquiring healthcare solutions:

I see that the unit supports the continuous development of our operations and that we need partners to solve our challenges. We can't solve everything here just on our own and we don't want to do it the way we've done before, trying to get a solution as close to our need as possible through a procurement process, but we want to influence the development of that solution so that it is as versatile as possible to meet our needs. (Innovation coordinator)

\section{Decisions}

Thus, in the first decision-making cycle the complexities observed by the hospital management were processed via three justification strategies into a decision to form an EU-funded project with the university of applied sciences and the city for establishing the testing unit. The cycle is summarized in Table 1, which describes the observed complexity in the first column, the justification strategies and how they were used in hiding the paradox of the decision in the second, and the decision made in the third.

<Insert: Table I. First sequence of the decision-making process.>

Although the decision to establish the testing unit reduced observed complexity, it simultaneously increased internal complexity that had to be reduced in the next decision-making cycle.

\subsection{Establishment of the testing unit}

\section{Complexity}

After the decision to establish the testing unit, increasing internal complexity was observed by hospital management in the form of practical problems to be solved. First, the testing activities and procedures for business collaboration had to be designed. Second, the hospital needed to position personnel to establish and run the new activities. Third, the testing unit's physical location and facilities needed to be decided upon.

\section{Justification}


By using the justification by problems strategy in the second decision-making cycle, the paradoxicality of decisions regarding the testing activities was concealed by businesses problems to be solved. The hospital undertook a survey with local businesses to discover their specific needs regarding the hospital's contribution to their product development and the results were used to justify the decisions regarding what types of testing services were offered to businesses.

Justification by experience strategy, meaning concealing the decision's paradox by the hospital's own or external examples, was used in decision-making regarding the testing unit's staffing and location. The quote below demonstrates how the hospital management referred to its previous experiences of development projects to justify a decision to hire a full-time manager for the unit.

(There was a) need for a coordinator (in the testing unit). One typical issue in (development projects) is that the excitement in the beginning and in the idea phase are fun. But when it comes to the serious work and the unpleasant parts of the work, bureaucracy and managing the bigger picture, the excitement might droop, and many projects have ended there. So, a professional is needed to execute the projects all the way. (Administrative specialist doctor)

The decision regarding the location of the new unit was in turn justified by external examples. The hospital management knew of problems in similar organizations abroad, which were located outside the hospital area and were difficult to reach. These external examples were referred to when deciding to locate the unit within the hospital's premises.

Justification by obligations strategy, meaning concealing a decision's paradox by emphasizing different function systems' demands, was also used to justify the decision regarding the testing unit's location. The unit's close location would enable the convenient participation of hospital's nurses and doctors in its activities and minimize the time spent on other than primary healthcare work. Hence, the location decision was justified as supporting fulfilling of the hospital's healthcare mandate. Also, a decision of a quite long testing service delivery time was also justified by the healthcare system's demands. The hospital needed to preserve enough time for the work arrangements of the wards to ensure enough staff on duty also during testing activities. Similarly, the hospital's healthcare purpose was the basis for deciding what types of products would be allowed for testing as is expressed in the following account.

--- The testing unit is primarily in the service of this hospital and there are detailed rules regarding what types (of products or services) can be brought in (for testing). They must be such that we estimate they could serve the operations of this hospital and add value to us. 
This is the ground rule and could be said to be the only one. (Hospital's development director)

In turn, decisions about the testing services content were justified by the legal system's demands: specific laws restricted involvement of patients and patient data in testing activities, as well as opportunities for clinical testing of medical devices.

\section{Decisions}

In the second decision-making cycle, internal complexity was reduced by decisions about the content and terms of the testing services, about the testing unit's location and its staffing. Referring to problems expressed by businesses, the hospital decided to create both physical and digital platforms for testing product prototypes and to provide feedback from healthcare professionals about the products for the businesses. Referring to the hospital's primary healthcare purpose, service delivery time was set up to six weeks, products that were not deemed to be beneficial to the hospital itself were excluded from testing and the unit was located and built into unused hospital facilities in the immediate environment of hospital wards. The location decision was also justified by examples from similar organization abroad. Referring to the hospital's legal obligations, patients and clinical tests were excluded from the testing activities, and only artificial patient data was used. Lastly, based on the hospital's previous experiences, a full-time manager for the testing unit was hired. Table II below summarizes the second cycle of the decision-making process.

<Insert: Table II. Second sequence of the decision-making process.>

\subsection{The testing unit's early operations}

\section{Complexity}

In the third decision-making cycle, the decisions made in the first and the second decision-making cycle led to increasing internal complexity. Specifically, the previous decision to establish the testing unit through the EU funded project caused problems to be solved by hospital management. Because the funding period was now ending, the unit's activities needed to be stabilized and financed in other ways.

\section{Justification}


The decision to continue the activities after EU funding had been processed from the beginning of the entire decision-making process. Justification by problems strategy, i.e. hiding the paradoxicality of the decision by problems the decision would solve, was used by referring to the hospital renewal project's continuing need for facilities and activities provided by the testing unit. Already at the start of the EU project, it was stated that the unit would become a permanent part of the hospital due to its benefits for the hospital's renewal project (Project plan 2014; EU funding application 2014). Justification by experience strategy, i.e. concealing the paradox by external or hospital's own examples, was used by referring to the hospital's and businesses' positive experiences gained during the EU-funded project. The data extract below from the project's final report illustrates how the justification by experience strategy was used by referring to the positive experiences of all stakeholders.

The most important stakeholders, businesses and healthcare professionals, are content with the improved possibilities for product and idea development related collaboration between public and private sector. Already during the project, businesses have received valuable feedback and references from healthcare professionals, speeding their product development. Healthcare professionals have advanced their ideas and innovation culture has been implemented in public healthcare. All in all, activity started at the project will produce better healthcare services for citizens in the future. (Project report 2017)

The last phrase of the quote above also demonstrates the use of justification by obligations strategy, i.e. concealing the paradox by function system's demands, by emphasizing the importance of the unit's continuation for developing future healthcare services, thus fulfilling the hospital's healthcare mandate. The strategy was also used to justify decisions regarding the unit's financing after the EU funding, as the legal obligations that the hospital as a public organization had to follow were referred to. The following interview extract by testing manager illustrates how the decision to finance the unit by charging businesses for the testing services was justified by the need to follow legislation excluding the option to self-finance them.

When we were still operating on EU project funding, we could invoice all our expenses from the project. Now, we need money from the businesses, but we have calculated the prices based on our operative expenses. We had a clear understanding from the beginning that we cannot offer testing free of charge, we cannot support businesses' product development with public funds. It's not possible. (Testing manager)

In addition to the three justification strategies described above, the third decision-making cycle differed from the previous two because now the hospital had also created a far-reaching decision premise which could be used in justifying the decision to make the activities permanent. The 
hospital district had renewed its organizational strategy, which acknowledged the testing unit as an integral part of the hospital's future development and its renewal project. For example, in an action plan developed after the EU project funding it could simply be stated that "The testing unit is in accordance with the hospital district's new strategy" (Action plan 2017) to justify the unit's continuation.

\section{Decisions}

Thus, via the justification strategies and the far-reaching decision premise, i.e. the hospital district's strategy, the observed internal complexity was absorbed by a decision to make the testing unit an official sub-organizational unit of the hospital, and to start charging businesses for the testing services to finance it. The third decision-making cycle is summarized in Table III.

<insert: Table III. Third sequence of the decision-making process.>

However, increasing complexity was still being observed by hospital management, but by the time of data collection, had not yet been processed in decision-making. By responding to the politicoeconomic expectations, the testing unit had formed a novel coupling between the hospital and the political and economic systems. Now increasing complexity produced by these systems was observed as problems to be solved. First, businesses were occasionally disappointed to the long service delivery time or when a product co-developed with the hospital was not taken into use due to the public tendering process. Second, national counterparts to the testing unit started emerging, which increased national competition and complicated attracting businesses, thus threatening the regional development goals of the health ecosystem. Additionally, there was internal complexity in terms of potential de-motivation by the staff to contribute to the activities of the testing unit stemming from the occasional disappointments in taking into use the co-developed products. Also, the hospital management perceived the unit's resourcing as being insufficient as the testing activities needed further development, and while the unit currently served mainly startups, the hospital wished to attract also bigger businesses into collaboration. Because the processing of these complexities had not yet resulted in any decisions, it marked a natural end of analyzing the present decision-making process. The next section discusses the contribution of the study to hybridity research, its limitations and practical limitations.

\section{Discussion and conclusion}




\subsection{Theoretical contribution to hybridity research}

This paper responds to the call for organizational level analysis of hybridity in healthcare (Denis et al., 2015; Powell and Castelli, 2017) by focusing on organizational decision-making in the context of a university hospital's hybridization. JJay (2013) argues that the paradoxical nature of hybrids stems from the involvement of contrasting institutional logics. By contrast, we view paradoxicality as an intrinsic part of all organizational decision-making and are interested in how organizations manage its tensions during hybridization. Hiding the paradox, i.e. the fact that a decision could be also made differently, is crucial for enhancing the acceptability and legitimacy of decisions, which is important in further decision-making. The paper's main theoretical contribution is the identification of three deparadoxization, or justification strategies, namely justification by problems, by experience and by obligations, which are deployed by decisionmakers in the absence of formal guidelines or rules to follow when making decisions regarding new organizational structures. In the case presented in this paper, when the decision-making process proceeded to its last cycle, the new structures and activities had been added to the official organizational strategy, potentially providing a basis for 'decidable decision-making premises' which could be used for justification of future decisions. Hence, the findings suggest that hybridization might require considerable efforts in organizational decision-making by mandating the development of several strategies for legitimizing decisions, before new organizational arrangements are distilled into formal decision-making premises.

Additionally, the paper advances a move beyond dualism inherent in many hybridity studies (e.g. Pache and Santos, 2013; Croft et al., 2015; McGivern et al., 2015; Broek et al., 2014) by exemplifying how decision-making during the hospital's hybridization combines the expectations of more than two institutional logics or societal function systems. In our case, it was the combined influence of the political and economic function systems that produced environmental complexity which was internalized in decision-making., However, demands of several other systems also had to be considered in each of the decision-making cycles. The 'justification by obligations' strategy was particularly important for translating the demands imposed by healthcare, education, science and legal function systems into legitimate decisions. Therefore, the findings suggest that hybridization of a well-established healthcare organization requires new organizational structures to be convincingly tied to the organization's existing societal purposes and obligations during 
decision-making processes. Due to the hospital's firmly established historical couplings to healthcare, science, education and law, premises for decisions oriented at other systems were less developed. By contrast, a 'born-hybrid' organization, such as a social enterprise, would presumably already from its establishment orient its far-reaching decision premises towards the separate function systems it chooses to couple.

As healthcare is a radically complex context with several function systems at play in organizations (Knudsen and Vogd, 2015), this complexity generates a wide variety of hybrid arrangements to be studied. In future research, it could be investigated how justification is achieved in decision-making unfolding in other types of healthcare organizations undergoing hybridization, or how justification develops in these organizations over time. The Luhmannian systems theoretical framework thus provides a fruitful alternative in the field of hybridity research.

\subsection{Limitations of the study}

The study is not without limitations. As we analyzed the decision-making process in retrospect via documents and interviews, it is possible that other justifications were also used but not written down in documents or remembered by the decision-makers during interviews. Moreover, as our analysis focused only on the decisions made and their justification, it is also possible that other decisions were made that did not get implemented, and thus appeared only as organizational noise (Luhmann, 1995) during the decision-making process. Identification of these unsuccessful decisions could have potentially enabled analysis of unsuccessful justification strategies. Had we been able to collect data by following the decision-making process more closely, for example by observational techniques at the time it was happening, the analysis could have captured also these aspects and thus provided an even richer depiction of the decision-making process.

\subsection{Practical implications}

Hybridization presents a challenge for decision-makers. Concealing the paradoxical nature of decisions oriented at new societal problems and unprecedented organizational structures can be laborious, as justifying decisions might require finding multiple grounds for each individual decision. The findings of this paper suggest that the ability to connect decisions to solving both societal and internal problems, to the organization's primary purposes and obligations, and to external and internal examples of similar activities, helps ensure decision acceptability and 
legitimacy in later decision-making. The findings also suggest that the inclusion of the new structures and activities in the organization's official strategies and plans, should help lessen the pressure in further decision-making. Decision-makers might also benefit from observing the organization's decision-making processes from the perspective of justification of decisions. Such an exploration might identify the types of strategies that are potentially unconsciously used and enable evaluation of their effectiveness. This could benefit future decision-making and situations in which desired new activities or structures face organizational resistance, by helping to reconsider how the proposed activities could be justified, i.e. how the paradoxicality of decisions could be hidden better.

\section{References}

Andersen, N. Å. and Born, A.W. (2007), "Heterophony and the postponed organization organizing autopoietic systems", Tamara: Journal for Critical Organization Inquiry, Vol. 6 No. 2, pp.176-186.

Baralou, E., Wolf, P. and Meissner, J. (2012), "Bright, excellent, ignored: The contribution of Luhmann's system theory and its problem of non-connectivity to academic management research", Historical Social Research, Vol. 37 No. 4, pp. 289-308.

Battilana, J., Besharov, M. and Mitzinneck, B. (2017), "On hybrids and hybrid organizing: A review and roadmap for future research", Greenwood, R., Oliver, R., Lawrence, T. and Meyer, R. (Ed.), The SAGE Handbook of Organizational Institutionalism, Sage, Los Angeles: Sage, pp. $128-163$.

Battilana, J. and Dorado, S. (2010), "Building sustainable hybrid organizations: The case of commercial microfinance organizations", Academy of Management Journal, Vol. 53 No. 6, pp. 1419-1440.

Battilana, J. and Lee, M. (2014), "Advancing research on hybrid organizing - Insights from the study of social enterprises", The Academy of Management Annals, Vol. 8 No. 1, pp. 397-441.

Besharov, M.L. and Smith, W.K. (2014), "Multiple institutional logics in organizations: explaining their varied nature and implications", Academy of Management Review, Vol. 39 No. 3, pp. 364381 .

Besio, C. and Pronzini, A. (2011), "Inside organization and out. Methodological tenets for empirical research inspired by systems theory", Historical Social Research, Vol. 36 No. 1, pp. $18-41$. 
Brandsen, T., Donk, W., and Putters, K. (2005), "Griffins or chameleons? Hybridity as a permanent and inevitable characteristic of the third sector", International Journal of Public Administration, Vol. 28 No. 9, pp. 749-765.

Byrkjeflot, H. and Jespersen, P.K. (2014), "Three conceptualizations of hybrid management in hospitals", International Journal of Public Sector Management, Vol. 27 No. 5, pp. 441-458.

Broek, J., Boselie, P. and Paauwe, J. (2014), "Multiple institutional logics in health care: 'Productive ward: Releasing time to care', Public Management Review, Vol. 16 No. 1, pp. 1-20

Croft, C., Currie, G. and Lockett, A. (2015), "Broken 'two-way windows'? An exploration of professional hybrids", Public Administration, Vol. 93 No. 2, pp. 380-394.

Denis, J.-L., Ferlie, E. and Van Gestel, N. (2015), "Understanding hybridity in public organizations", Public Administration, Vol. 93 No. 2, pp. 273-289.

DiMaggio, P.J. (1991), “Constructing an organizational field as a professionalization project: U.S. art museums, 1920-1940”, Powell, W.W. and DiMaggio, P. (Ed.), The New Institutionalism in Organizational Analysis, University of Chicago Press, Chicago, IL, pp. 267-292.

Ferreira, S. (2014), "Sociological observation of the third sector through systems theory: An analytical proposal", Voluntas, Vol. 25, pp. 1671-1693.

Friedland, R. and Alford, R.R. (1991), "Bringing society back in: symbols, practices, and institutional contradictions", Powell, W.W. and DiMaggio, P. (Ed), The New Institutionalism in Organizational Analysis, University of Chicago Press, Chicago, IL, pp. 232-263.

Gestel, N., Denis, J-L. \& Ferlie, E. (2020), "Hybridity in public organisations", Billis, D. and Rochester, C. (Ed), Handbook on Hybrid Organisations, Edward Elgar Publishing, Cheltenham, UK, Northhampton, MA, pp. 48-65.

Gulbrandsen, M. (2011), "Research institutes as hybrid organizations: central challenges to their legitimacy”, Policy Science, Vol. 44, pp. 215-230.

Hernes, T. and Bakken, T. (2003), "Implications of self-reference: Niklas Luhmann's Autopoiesis and organization theory”, Organization Studies, Vol. 24 No. 9, pp. 1511-1535.

Jay, J. (2013), "Navigating paradox as a mechanism of change and innovation in hybrid organizations", Academy of Management Journal, Vol. 56 No. 1, pp. 137-159.

Knudsen, M. (2006), "Displacing the paradox of decision-making”, Seidl, D. and Becker, K.H. (Ed.) Niklas Luhmann and Organization Studies, CBS Press, Copenhagen, pp. 107-126.

Knudsen, M. (2007), "Structural couplings between organizations and function systems - looking at standards in health care", Cybernetics and Human Knowing, Vol. 14 No. 1, pp. 111-131. 
Knudsen, M. (2011), "Surprised by method - functional method and systems theory", Historical Social Research, Vol. 36 No. 1, pp. 124 - 142.

Knudsen, M. and Vogd, W. (2015), "Introduction: Health care, systems theory and polycontexturality", Knudsen, M and Vogd, W. (Ed.) Systems theory and the sociology of health and illness - Observing health care, Routledge, New York and London, pp. 1-22.

Luhmann, N. (1986) Ökologische Kommunikation. Opladen, Wesdeutscher Verlag.

Luhmann, N. (1995), Social Systems. Stanford University Press, Stanford.

Luhmann, N. (1997), "Limits of steering”, Theory, Culture \& Society, Vol. 14 No. 1, pp. 41-57.

Luhmann, N. (2018), Organization and Decision. Cambridge University Press, Cambridge.

Mair, J., Mayer, J. and Lutz, E. (2015), "Navigating institutional plurality: organizational governance in hybrid organizations", Organization Studies, Vol. 36 No. 6, pp. 713-739.

McDermott, A.M., Hamel, L.M., Steel, D., Flood, P.C. and Mkee, L. (2015), "Hybrid healthcare governance for improvement? Combining top-down and bottom-up approaches to public sector regulation", Public Administration, Vol. 93 No. 2, pp. 324-344

Murray, F. (2010), "The oncomouse that roared: Hybrid exchange strategies as a source of distinction at the boundary of overlapping institutions", American Journal of Sociology, Vol. 116 No. 2, pp. 341-388.

Nassehi, A. (2005), “Organizations as decision machines: Niklas Luhmann's theory of organized social systems”, The Sociological Review, Vol. 53 No. 1, pp. 178-191.

Pache, A. and Santos, F. (2010), "When worlds collide: The internal dynamics of organizational responses to conflicting institutional demands", Academy of Management Review, Vol. 35, pp. $455-476$.

Pache, A. and Santos, F. (2013), "Inside the hybrid organization: Selective coupling as a response to competing institutional logics", Academy of Management Journal, Vol. 56 No. 4, pp. 9721001 .

Powell, M. and Castelli, M. (2017), “"Strange animals": hybrid organisations in health care”, Journal of Health Organization and Management, Vol. 31 No. 7, pp. 746-762.

Schneider, A., Wickert, C. and Marti, E. (2017), "Reducing complexity by creating complexity: A systems theory perspective on how organizations respond to their environments", Journal of Management Studies, Vol. 54 No. 2, pp. 182-208. 
Seidl, D. (2006), "The basic concepts of Luhmann's theory of social systems", Seidl, D. and Becker, K.H. (Ed.) Niklas Luhmann and organization studies, Liber \& Copenhagen Business School Press, Copenhagen, pp. 21-53.

Seidl, D. and Becker, K.H. (2006a), (Eds.) Niklas Luhmann and Organization Studies, Liber \& Copenhagen Business School Press, Copenhagen.

Seidl, D. and Becker, K.H. (2006b), "Organizations as Distinction Generating and processing systems: Niklas Luhmann's contribution to organization studies”, Organization, Vol. 13 No. 1, pp. 1350-5084.

Seidl, D. and Mormann, H. (2015), "Niklas Luhmann as organization theorist", Adler, P., Du Gay, P. and Reed, M. (Ed.) Oxford Handbook of Sociology, Social Theory and Organization Studies: Contemporary Currents, Oxford University Press, Oxford, pp. 125-157.

Singh, P. and Butler, S.M. (2015), "Intermediaries in integrated approaches to health and economic mobility". B Economic Studies at Brookings, A Series of Discussion Papers on Building Healthy Neighborhoods No. 4. Washington, DC, The Brookings Institution.

Skelcher, C. and Smith, S. (2015), “Theorizing hybridity: Institutional logics, complex organizations, and actor identities: The case of nonprofits", Public Administration Vol. 93 No. 2, pp. 433-448.

Spyridonidis, D., Hendy, J. and Barlow, J. (2015), "Understanding hybrid roles: The role of identity processes amongst physicians", Public Administration, Vol. 93 No. 2, pp. 395-411.

Stichweh, R. (1999) The form of the university. In D. Baecker (Ed.) Problems of Form. Stanford, Stanford University Press.

Thornton, P.H., Ocasio, W. and Lounsbury, M. (2012), The Institutional Logics Perspective: A New Approach to Culture, Structure and Process, Oxford University Press, Oxford.

Waring, J. (2015), "Mapping the public sector diaspora: Towards a model of inter-sectoral cultural hybridity using evidence from the English healthcare reforms", Public Administration, Vol 93. No. 2, pp. 345-362. 